\title{
SEARCHING FOR SUPERSYMMETRIC DARK MATTER. THE DIRECTIONAL RATE AND THE MODULATION EFFECT DUE TO CAUSTIC RINGS .
}

\author{
J. D. VERGADOS \\ Theoretical Physics Section, University of Ioannina, GR-45110, Greece \\ E-mail:Vergados@cc.uoi.gr
}

\begin{abstract}
The detection of the theoretically expected dark matter is central to particle physics and cosmology. Current fashionable supersymmetric models provide a natural dark matter candidate which is the lightest supersymmetric particle (LSP). The allowed parameter space of such models combined with fairly well understood physics (quark substructure of the nucleon and nuclear structure) permit the evaluation of the event rate for LSP-nucleus elastic scattering. The thus obtained event rates, which sensitively depend on the allowed parameter space parameters, are usually very low or even undetectable. So, for background reduction, one would like to exploit two nice features of the reaction, the directional rate, which depends on the sun's direction of motion and the modulation effect, i.e. the dependence of the event rate on the earth's annual motion. In the present paper we study these phenomena in a specific class of non isothermal models, which take into account the late in-fall of dark matter into our galaxy, producing flows of caustic rings. We find that the modulation effect arising from such models is smaller than that found previously with isothermal symmetric velocity distributions and much smaller compared to that obtained using a realistic asymmetric distribution with enhanced dispersion in the galactocentric direction.
\end{abstract}

\section{Introduction}

In recent years the consideration of exotic dark matter has become necessary in order to close the Universe 1, 2. Furthermore in in order to understand the large scale structure of the universe it has become necessary to consider matter made up of particles which were non-relativistic at the time of freeze out. This is the cold dark matter component (CDM). The COBE data suggest that $\mathrm{CDM}$ is at least $60 \%$. On the other hand during the last few years evidence has appeared from two different teams the High-z Supernova Search Team 0 and the Supernova Cosmology Project 6 , which suggests that the Universe may be dominated by the cosmological constant $\Lambda \mathrm{r}$. As a matter of fact recent data the situation can be adequately described by a barionic component $\Omega_{B}=0.1$ along with the exotic components $\Omega_{C D M}=0.3$ and

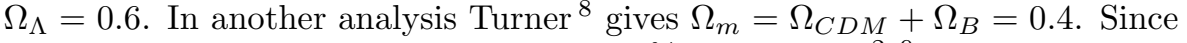
the non exotic component cannot exceed $40 \%$ of the CDM 2, $\mathrm{Q}$, there is room for the exotic WHMP's (Weakly Interacting Massive Particles). In fact the DAMA experiment 10 has claimed the observation of one signal in direct detection of 
a WIMP, which with better statistics has subsequently been interpreted as a modulation signal 11 .

The above developments are in line with particle physics considerations. Thus, in the currently favored supersymmetric (SUSY) extensions of the standard model, the most natural WIMP candidate is the LSP, i.e. the lightest supersymmetric particle. In the most favored scenarios the LSP can be simply described as a Majorana fermion, a linear combination of the neutral components of the gauginos and Higgsinos $1,2-21$.

Since this particle is expected to be very massive, $m_{\chi} \geq 30 \mathrm{GeV}$, and extremely non relativistic with average kinetic energy $T \leq 100 \mathrm{KeV}$, it can be directly detected $12-13$ mainly via the recoiling of a nucleus $(\mathrm{A}, \mathrm{Z})$ in the elastic scattering process:

$$
\chi+(A, Z) \rightarrow \chi+(A, Z)^{*}
$$

( $\chi$ denotes the LSP). In order to compute the event rate needs the following ingredients:

1) An effective Lagrangian at the quark level in the context of supersymmetry as described in Refs. 3 , Bottino et al. 18 and 11 .

2) A a quark model for the nucleon, needed in going from the quark to the nucleon level, since the gatained results are sensitive to the presence of quarks other than $u$ and d 14.22, 23 .

3) Compute the differentialcross sections using as reliable as possible many body nuclear wave functions. $24-27$.

The obtained rates sensitively depend on the input from the allowed SUSY parameter space. Since the expected rates are extremely low or even undetectable with present techniques, one would like to exploit the characteristic signatures provided by the reaction. Such are: a) The modulation effect, i.e the dependence of the event rate on the velocity of the Earth and b) The directional event rate, which depends on the velocity of the sun around the galaxy as wel_as the the velocity of the Earth. The latter effect, recognized sometime agg 28 has recently begun to appear feasible by the planned UKDMC experiment 29. We will study both of these effects in the present work.

In our previous letter 16 and its subsequent expanded version 17 we found enhanced modulation, if one uses an appropriate asymmetric velocity distribution with enhanced dispersion in the galactocentric direction 31 .

The isolated galaxies are, however, surrounded by cold dark matter which, due to gravity, keeps falling continuously on them from all directions 32 . As a result one has caustic rings with matter density, which depends on space and velocity.

It is the purpose of our present paper to exploit the results of such a scenario and calculate quantitavely the resulting modulation effect in the usual 
(differential and total ) rate for LSP-nucleus elastic scattering. We will alsp study the directional rates in the spirit of the recent work of Copi et al 30 . For the reader's convenience we will give a very brief description of the basic ingredients on how to calculate LSP-nucleus scattering cross section. We will not, however, elaborate on how one gets the needed parameters from supersymmetry. The calculation of these parameters has become pretty standard. One starts with representative input in the restricted SUSY parameter space as described in the literafure, e.g. Bottino et al. 18, Kane et al. , Castano et al. and Arnowitt et al. 19 .

After this we will specialize our study in the case of the nucleus ${ }^{127} I$, which is one of the most popular targets 103334 . We will present our results a function of the LSP mass, $m_{\chi}$, in a way which can be easily understood by the experimentalists.

\section{The Basic Ingredients for LSP Nucleus Scattering}

Because of lack of space we are not going to elaborate here further on the construction of the effective Lagrangian derived from supersymmetry, but refer the reader to the literature $12,13,15,1836$. For the reader's convenience we will summarize our previous 4 formulas related to the LSP-Nucleus cross section and the event rates. The effective Lagrangian can be obtained in first order via Higgs exchange, s-quark exchange and Z-exchange. In a formalism familiar from the theory of weak interactions we write

$$
L_{e f f}=-\frac{G_{F}}{\sqrt{2}}\left\{\left(\bar{\chi}_{1} \gamma^{\lambda} \gamma_{5} \chi_{1}\right) J_{\lambda}+\left(\bar{\chi}_{1} \chi_{1}\right) J\right\}
$$

where

$$
J_{\lambda}=\bar{N} \gamma_{\lambda}\left(f_{V}^{0}+f_{V}^{1} \tau_{3}+f_{A}^{0} \gamma_{5}+f_{A}^{1} \gamma_{5} \tau_{3}\right) N
$$

and

$$
J=\bar{N}\left(f_{s}^{0}+f_{s}^{1} \tau_{3}\right) N
$$

We have neglected the uninteresting pseudoscalar and tensor currents. Note that, due to the Majorana nature of the LSP, $\bar{\chi}_{1} \gamma^{\lambda} \chi_{1}=0$ (identically). The parameters $f_{V}^{0}, f_{V}^{1}, f_{A}^{0}, f_{A}^{1}, f_{S}^{0}, f_{S}^{1}$ depend on the SUSY model employed. In SUSY models derived from minimal SUGRA the allowed parameter space is characterized at the GUT scale by five parameters, two universal mass parameters, one for the scalars, $m_{0}$, and one for the fermions, $m_{1_{1} 2}$, as well as

the parameters $\tan \beta$, one of $A_{0}$ (or $m_{t}^{\text {pole }}$ ) and the sign of $\mu$. 19 . Deviations from universality at the GUT scale have also been considered and found useful 
20. We will not elaborate further on this point since the above parameters involving universal masses have already been computed in some models 1236 and effects resulting from deviations from universality will be found elsewhere (see Arnowitt et al in Ref. 20 and Bottino et al in Ref. 18). For some choices in the allowed parameter space the obtained couplings can be found in a previous paper 36 .

With the above ingredients the differential cross section can be cast in the form

$$
\begin{gathered}
d \sigma(u, v)=\frac{d u}{2\left(\mu_{r} b v\right)^{2}}\left[\left(\bar{\Sigma}_{S}+\bar{\Sigma}_{V} \frac{v^{2}}{\left\langle v^{2}>\right.}\right) F^{2}(u)+\bar{\Sigma}_{s p i n} F_{11}(u)\right] \\
\bar{\Sigma}_{S}=\sigma_{0}\left(\frac{\mu_{r}}{m_{N}}\right)^{2}\left\{A^{2}\left[\left(f_{S}^{0}-f_{S}^{1} \frac{A-2 Z}{A}\right)^{2}\right] \simeq \sigma_{p, \chi^{0}}^{S} A^{2}\left(\frac{\mu_{r}}{\mu_{r}(N)}\right)^{2}\right. \\
\bar{\Sigma}_{s p i n}=\sigma_{p, \chi^{0}}^{\text {spin }} \zeta_{\text {spin }} \\
\left.\zeta_{\text {spin }}=\frac{\left(\mu_{r} / \mu_{r}(N)\right)^{2}}{3\left(1+\frac{f_{A}^{0}}{f_{A}^{1}}\right)^{2}}\left[\left(\frac{f_{A}^{0}}{f_{A}^{1}} \Omega_{0}(0)\right)^{2} \frac{F_{00}(u)}{F_{11}(u)}+2 \frac{f_{A}^{0}}{f_{A}^{1}} \Omega_{0}(0) \Omega_{1}(0) \frac{F_{01}(u)}{F_{11}(u)}+\Omega_{1}(0)\right)^{2}\right] \\
\bar{\Sigma}_{V}=\frac{\left(\mu_{r} / \mu_{r}(N)\right)^{2}}{\left(1+\frac{f_{V}^{1}}{f_{V}^{0}}\right)^{2}} A^{2}\left(1-\frac{f_{V}^{1}}{f_{V}^{0}} \frac{A-2 Z}{A}\right)^{2} \frac{<v^{2}>}{c^{2}}\left[1-\frac{1}{\left(2 \mu_{r} b\right)^{2}} \frac{2 \eta+1}{(1+\eta)^{2}} \frac{\langle 2 u\rangle}{\left\langle v^{2}\right\rangle}\right]
\end{gathered}
$$

$\sigma_{p, \chi^{0}}^{i}=$ proton cross-section, $i=S$, spin, $V$ given by:

$\sigma_{p, \chi^{0}}^{S}=\sigma_{0}\left(f_{S}^{0}\right)^{2}$ (scalar), (the isovector scalar is negligible, i.e. $\sigma_{p}^{S}=\sigma_{n}^{S}$ )

$\sigma_{p, \chi^{0}}^{\text {spin }}=\sigma_{0} 3\left(f_{A}^{0}+f_{A}^{1}\right)^{2}(\operatorname{spin}), \sigma_{p, \chi^{0}}^{V}=\sigma_{0}\left(f_{V}^{0}+f_{V}^{1}\right)^{2}$ (vector)

where $m_{p}$ is the proton mass, $\eta=m_{x} / m_{N} A, \mu_{r}$ is the LSP-nucleus reduced mass , $\mu_{r}(N)$ is the LSP-nucleon reduced mass and

$$
\sigma_{0}=\frac{1}{2 \pi}\left(G_{F} m_{N}\right)^{2} \simeq 0.77 \times 10^{-38} \mathrm{~cm}^{2}
$$


with

$$
u=q^{2} b^{2} / 2 \quad \text { or } \quad Q=Q_{0} u, \quad Q_{0}=\frac{1}{A m_{N} b^{2}}
$$

where $\mathrm{b}$ is (the harmonic oscillator) size parameter, $\mathrm{q}$ is the momentum transfer and $\mathrm{Q}$ is the energy transfer to the nucleus

In the above expressions $F(u)$ is the nuclear form factor and

$$
F_{\rho \rho^{\prime}}(u)=\sum_{\lambda, \kappa} \frac{\Omega_{\rho}^{(\lambda, \kappa)}(u)}{\Omega_{\rho}(0)} \frac{\Omega_{\rho^{\prime}}^{(\lambda, \kappa)}(u)}{\Omega_{\rho^{\prime}}(0)}, \quad \rho, \rho^{\prime}=0,1
$$

are the spin form factors 13 ( $\rho, \rho^{\prime}$ are isospin indices) Both form factors are normalized to one at $u=0 . \Omega_{0}\left(\Omega_{1}\right)$ are the static isoscalar (isovector) spin matrix elements.

The non-directional event rate is given by:

$$
R=R_{n o n-d i r}=\frac{d N}{d t}=\frac{\rho(0)}{m_{\chi}} \frac{m}{A m_{N}} \sigma(u, v)|v|
$$

Where $\rho(0)=0.3 \mathrm{GeV} / \mathrm{cm}^{3}$ is the LSP density in our vicinity and $\mathrm{m}$ is the detector mass

The differential non-directional rate can be written as

$$
d R=d R_{n o n-d i r}=\frac{\rho(0)}{m_{\chi}} \frac{m}{A m_{N}} d \sigma(u, v)|v|
$$

where $d \sigma(u, v)$ was given above.

The directional differential rate 30 in the direction $\hat{e}$ is given by :

$$
d R_{d i r}=\frac{\rho(0)}{m_{\chi}} \frac{m}{A m_{N}} v \cdot \hat{e} H(v \cdot \hat{e}) \frac{1}{2 \pi} d \sigma(u, v)
$$

where $H$ the Heaviside step function. The factor of $1 / 2 \pi$ is introduced, since the differential cross section of the last equation is the same with that entering the non-directional rate, i.e. after an integration over the azimuthal angle around the nuclear momentum has been performed. In other words, crudely speaking, $1 /(2 \pi)$ is the suppression factor we expect in the directional rate compared to the usual one. The precise suppression factor depends, of course, on the direction of observation. 


\section{Convolution of the Event Rate}

We have seen that the event rate for LSP-nucleus scattering depends on the relative LSP-target velocity. In this section we will examine the consequences of the earth's revolution around the sun (the effect of its rotation around its axis is expected to be negligible) i.e. the modulation effect. In the past this has been accomplished by assuming a consistent LSP velocity dispersion, such as a Maxwell distribution or asymmetric velacity distribution with enhanced dispersion in the galactocentric direction 31] 16, 17. More recently other very interesting non-isothermal approaches have been proposed, which consider the in-fall of dark matter into the galaxy producing flows of caustic rings. In particular the predictions of a self-similar model have been put forward as a possible scenario for dark matter density-velocity distribution, see e.g. Sikivie et al 32. The implications of such distributions on the direct detection rates and, in particular, on the directional ones and the modulation effect are the subject of this work. Before proceeding furtyher we should mention that after our manuscript had been prepared another approach has been suggested, which links the distribution to the density profile via Eddigton's formula 3 .

Following Sikivie we will consider $2 \times N$ caustic rings, $(\mathrm{i}, \mathrm{n}), \mathrm{i}=(+.-)$ and $\mathrm{n}=1,2, \ldots \mathrm{N}(\mathrm{N}=20$ in the model of Sikivie et al), each of which contributes to the local density a fraction $\bar{\rho}_{n}$ of the of the summed density $\bar{\rho}$ of each of the $i=+,-$. It contains WIMP like particles with velocity $\mathbf{y}_{n}^{\prime}=\left(y_{n x}^{\prime}, y_{n y}^{\prime}, y_{n z}^{\prime}\right)$ in units of essentially the sun's velocity $\left(v_{0}=220 \mathrm{Km} / \mathrm{s}\right)$, with respect to the galactic center. The z-axis is chosen in the direction of the disc's rotation, i.e. in the direction of the motion of the the sun, the $y$-axis is perpendicular to the plane of the galaxy and the $\mathrm{x}$-axis is in the radial direction. We caution the reader that these axes are traditionally indicated by astronomers as $\hat{e}_{\phi}, \hat{e}_{r}, \hat{e}_{e}$ respectively. The needed quantities are taken from the work of Sikivie et al 32 (see in TABLE I), via the definitions

$$
y_{n}^{\prime}=v_{n} / v_{0}, y_{n z}^{\prime}=v_{n \phi} / v_{0}=y_{n z}, y_{n x}^{\prime}=v_{n r} / v_{0}=y_{n x}, y_{n y}^{\prime}=v_{n z} / v_{0}=
$$

$y_{n y}, \rho_{n}=d_{n} / \bar{\rho}, \bar{\rho}=\sum_{n=1}^{N} d_{n}$ and $y_{n}=\left[\left(y_{n z}-1\right)^{2}+y_{n y}^{2}+y_{n x}^{2}\right]^{1 / 2}$ (for each flow +.-). This leads to a velocity distribution of the form:

$$
f\left(v^{\prime}\right)=\sum_{n=1}^{N} \delta\left(v^{\prime}-v_{0} \mathbf{y}_{n}^{\prime}\right)
$$

Since the axis of the ecliptic 33 lies very close to the $y, z$ plane the velocity of the earth around the sun is given by

$$
v_{E}=v_{0}+v_{1}=v_{0}+v_{1}(\sin \alpha \hat{\mathbf{x}}-\cos \alpha \cos \gamma \hat{\mathbf{y}}+\cos \alpha \sin \gamma \hat{\mathbf{z}})
$$

where $\alpha$ is the phase of the earth's orbital motion, $\alpha=2 \pi\left(t-t_{1}\right) / T_{E}$, where $t_{1}$ is around second of June and $T_{E}=1$ year. 
One can now express the above distribution in the laboratory frame 17 by writing $v^{\prime}=v+v_{E}$

\section{Expressions for the Differential Event Rate.}

The mean value of the non-directional event rate of Eq. (15), is given by

$$
\left\langle\frac{d R}{d u}\right\rangle=\frac{\rho(0)}{m_{\chi}} \frac{m}{A m_{N}} \int f\left(v, v_{E}\right)|v| \frac{d \sigma(u, v)}{d u} d^{3} v
$$

The above expression can be more conveniently written as

$$
\left\langle\frac{d R}{d u}\right\rangle=\frac{\rho(0)}{m_{\chi}} \frac{m}{A m_{N}} \sqrt{\left\langle v^{2}\right\rangle}\left\langle\frac{d \Sigma}{d u}\right\rangle
$$

where

$$
\left\langle\frac{d \Sigma}{d u}\right\rangle=\int \frac{|v|}{\sqrt{\left\langle v^{2}\right\rangle}} f\left(v, v_{E}\right) \frac{d \sigma(u, v)}{d u} d^{3} v
$$

There are now experiments under way aiming at measuring directional rates 29 using TPC counters which permit the observation of the recoiling nucleus is observed in a certain direction. From a theoretical point of view the directional rates have been previously discussed by Spergel 28 and Copi et al 30. The mean value of the directional differential event rate of Eq. (16), is defined by

$$
\left\langle\frac{d R}{d u}\right\rangle_{d i r}=\frac{\rho(0)}{m_{\chi}} \frac{m}{A m_{N}} \frac{1}{2 \pi} \int f\left(v, v_{E}\right) v . \hat{e} H(v . \hat{e}) \frac{d \sigma(u, v)}{d u} d^{3} v
$$

where $\hat{\mathbf{e}}$ is the unit vector in the direction of observation. It can also be more conveniently expressed as

$$
\left\langle\frac{d R}{d u}\right\rangle_{d i r}=\frac{\rho(0)}{m_{\chi}} \frac{m}{A m_{N}} \sqrt{\left\langle v^{2}\right\rangle}\left\langle\frac{d \Sigma}{d u}\right\rangle_{d i r}
$$

where

$$
\left\langle\frac{d \Sigma}{d u}\right\rangle_{d i r}=\frac{1}{2 \pi} \int \frac{v \cdot \hat{e} H(v \cdot \hat{e})}{\sqrt{\left\langle v^{2}\right\rangle}} f\left(v, v_{E}\right) \frac{d \sigma(u, v)}{d u} d^{3} v
$$

It is clear that the rate will depend on the direction of observation, showing a strong correlation with the direction of the sun's motion. In a favorable situation the rate will merely be suppressed by a factor of $2 \pi$ relative to the non-directional rate. We will specialize our results in the case of caustic rings. 


\section{A. The Non-directional Differential Event Rate in the Case of caustic Rings}

Eq. 21 takes the form

$$
\left\langle\frac{d \Sigma}{d u}\right\rangle=\frac{2 \bar{\rho}}{\rho(0)} a^{2}\left[\bar{\Sigma}_{S} \bar{F}_{0}(u)+\frac{\left\langle v^{2}\right\rangle}{c^{2}} \bar{\Sigma}_{V} \bar{F}_{1}(u)+\bar{\Sigma}_{\text {spin }} \bar{F}_{\text {spin }}(u)\right]
$$

We remind the reader that $\bar{\rho}$ was obtain for each type of flow (+ or -), which explains the factor of two. In the Sikivie model 32 we have $(2 \bar{\rho} / \rho(0) \simeq 1.0$, i.e. the whole dark matter density lies in the form of caustic rings. In hybrid models, which contain in addition an isothermal component, it is only a fraction, since the sum of all densities should be $\rho(0)$.

The quantities $\bar{\Sigma}_{i}, i=S, V$, spin are given by Eqs. (6)- (9). The quantities $\bar{F}_{0}, \bar{F}_{1}, \bar{F}_{\text {spin }}$ are obtained from the corresponding form factors via the equations

$$
\begin{gathered}
\bar{F}_{k}(u)=F^{2}(u) \bar{\Psi}_{k}(u) \frac{(1+k)}{2 k+1} \quad, \quad k=0,1 \\
\bar{F}_{\text {spin }}(u)=F_{11}(u) \bar{\Psi}_{0}(u)
\end{gathered}
$$

The functions $\tilde{\Psi}_{k}(u)$ depend on the model. Introducing the parameter

$$
\delta=\frac{2 v_{1}}{v_{0}}=0.27
$$

we find in the case of Sikivie model

$$
\tilde{\Psi}_{k}(u)=\sqrt{\frac{2}{3}} \sum_{n=1}^{N} \bar{\rho}_{n} \tilde{y}_{n}^{2(k-1)} \Theta\left(\frac{\tilde{y}_{n}^{2}}{a^{2}}-u\right) \tilde{y_{n}}
$$

with

$$
a=\frac{1}{\sqrt{2} \mu_{r} b v_{0}}
$$

and

$\tilde{y_{n}}=\left[\left(y_{n z}-1-\frac{\delta}{2} \sin \gamma \cos \alpha\right)^{2}+\left(y_{n y}+\frac{\delta}{2} \cos \gamma \cos \alpha\right)^{2}+\left(y_{n x}-\frac{\delta}{2} \sin \alpha\right)^{2}\right]^{1 / 2}$

The above expressions (25)-(27) depend not only on $u$, but on the phase of the Earth as well as in the angle $\gamma \simeq \pi / 2$. If, however, in the $\Theta$-function we approxmate $\tilde{y}_{n}$ by $y_{n}$ given by 


$$
\left.y_{n}=\left[\left(y_{n z}-1\right)\right)^{2}+y_{n y}^{2}+y_{n x}^{2}\right]^{1 / 2}
$$

i.e. neglect threshold effects originating from the motion of the Earth, the obtained expressions can be simplified. In fact, if we then expand $\tilde{\Psi}_{k}(u)$ in the small parameter $\delta$ and keep terms up to linear in it, the dependence on the phase of the Earth can be seperated from the dependence on $u$. Thus, to an approximation which is valid to no worse than $10 \%$ for all $u$, the non-directional differential rate takes the form

$$
\left\langle\frac{d R}{d u}\right\rangle=\bar{R} \frac{2 \bar{\rho}}{\rho(0)} t T(u)[1-\cos \alpha H(u)]
$$

where now $T(u)$ and $H(u)$ are now function of the variable $u$.

The last expression must be compared to that of isothermal models:

$$
\left\langle\frac{d R}{d u}\right\rangle=\bar{R} \frac{\rho^{\prime}(0)}{\rho(0)} t T(u)[1+\cos \alpha H(u)]
$$

with $\rho^{\prime}(0)$ being the density associated with the isothermal mode. In hybrid models it is less than $\rho(0)$. In the above expressions $\bar{R}$ is the rate obtained in the conventional approach 12 by neglecting the folding with the LSP velocity and the momentum transfer dependence of the differential cross section, i.e. by

$$
\bar{R}=\frac{\rho(0)}{m_{\chi}} \frac{m}{A m_{N}} \sqrt{\left\langle v^{2}\right\rangle}\left[\bar{\Sigma}_{S}+\bar{\Sigma}_{\text {spin }}+\frac{\left\langle v^{2}\right\rangle}{c^{2}} \bar{\Sigma}_{V}\right]
$$

where $\bar{\Sigma}_{i}, i=S, V$, spin have been defined above, see Eqs (6) - (9).

The factor $T(u)$ takes care of the u-dependence of the unmodulated differential rate. It is defined so that

$$
\int_{u_{\min }}^{u_{\max }} d u T(u)=1
$$

i.e. it is the relative differential rate. $u_{\min }$ is determined by the energy cutoff due to the performance of the detector,i.e

$$
u_{\min }=\frac{Q_{\min }}{Q_{0}}
$$

while $u_{\max }$ is determined the via the relations:

$$
u_{\max }=\min \left(\frac{y_{e s c}^{2}}{a^{2}}, \max \left(\frac{y_{n}^{2}}{a^{2}}\right), n=1,2, \ldots, N\right)
$$


On the other hand $H(u)$ gives the energy transfer dependent modulation amplitude (relative to the unmodulated amplitude). The quantity $t$ takes care of the modification of the total rate due to the nuclear form factor and the folding with the LSP velocity distribution. Since the functions $\bar{F}_{0}(u), \bar{F}_{1}$ and $\bar{F}_{\text {spin }}$ have a different dependence on $\mathrm{u}$, the functions $T(u)$ and $H(u)$ and $t$, in principle, depend somewhat on the SUSY parameters. If, however, we ignore the small vector contribution and assume (i) the scalar and axial (spin) dependence on $\mathrm{u}$ is the same, as seems to be the case for light systems 3839 , or (ii) only one mechanism (S, V, spin) dominates, the parameter $\bar{R}$ contains the dependence on all SUSY parameters. The parameters $t$ and $T(u)$ depend on the LSP mass and the nuclear parameters, while the $H(u)$ depends only on the parameter $a$. Eq. 21 takes the form

\section{B. Expressions for the Directional Differential Event Rate}

The model of Sikivie et al 32 , which is not spherically symmetric, offers itself as a perfect example for the study of the directional rates. Working as in the previous section we get 17

$$
\left\langle\frac{d \Sigma}{d u}\right\rangle_{d i r}=\frac{2 \bar{\rho}}{\rho(0)} \frac{a^{2}}{2 \pi}\left[\bar{\Sigma}_{S} F_{0}(u)+\frac{\left\langle v^{2}\right\rangle}{c^{2}} \bar{\Sigma}_{V} F_{1}(u)+\bar{\Sigma}_{\text {spin }} F_{\text {spin }}(u)\right]
$$

where the $\bar{\Sigma}_{i}, i=S, V$, spin are given by Eqs. (5)- (9). The quantities $F_{0}, F_{1}, F_{\text {spin }}$ are obtained from the equations

$$
\begin{gathered}
F_{k}(u)=F^{2}(u) \Psi_{k}(u) \frac{(1+k)}{2 k+1}, k=0,1 \\
F_{\text {spin }}(u)=F_{11}(u) \Psi_{0}(u)
\end{gathered}
$$

In the Sikivie model we find

$$
\begin{aligned}
\Psi_{k}(u) & =\sqrt{\frac{2}{3}} \sum_{n=1}^{N} \bar{\rho}_{n} \tilde{y}_{n}^{2(k-1)} \Theta\left(\frac{y_{n}^{2}}{a^{2}}-u\right) \mid\left(y_{n z}-1-\frac{\delta}{2} \sin \gamma \cos \alpha\right) \mathbf{e}_{z} \cdot \mathbf{e} \\
& +\left(y_{n y}+\frac{\delta}{2} \cos \gamma \cos \alpha\right) \mathbf{e}_{y} \cdot \mathbf{e}+\left(y_{n x}-\frac{\delta}{2} \sin \alpha\right) \mathbf{e}_{x} \cdot \mathbf{e} \mid
\end{aligned}
$$

In the model considered here the z-component of the LSP velocity, with respect to the galactic center, for some rings is smaller than the sun's velocity, while for some others it is larger. The components in the $\mathrm{y}$ and the $\mathrm{x}$ directions are opposite for the + and - flows. So we will distinguish the following cases: a) $\hat{e}$ has a component in the sun's direction of motion, i.e. $0<\theta<\pi / 2$, labeled by 
$\mathrm{u}$ (up). b) Detection in the direction specified by $\pi / 2<\theta<\pi$, labeled by $\mathrm{d}$ (down). The differential directional rate takes a different form depending on which quadrant the observation is made. Thus, keeping terms up to linear in $\delta$, we find:

1. In the first quadrant (azimuthal angle $0 \leq \phi \leq \pi / 2$ ).

$$
\begin{aligned}
\left\langle\frac{d R^{i}}{d u}\right\rangle & =\bar{R} \frac{2 \bar{\rho}}{\rho(0)} \frac{t}{2 \pi} T(u)\left[\left(R_{z}^{i}(u)-\cos \alpha H_{1}^{i}(u)\right)\left|\mathbf{e}_{z} \cdot \mathbf{e}\right|\right. \\
& +\left(R_{y}^{i}+\cos \alpha H_{2}^{i}(u)+\frac{H_{c}^{i}(u)}{2}(|\cos \alpha|+\cos \alpha)\right)\left|\mathbf{e}_{y} \cdot \mathbf{e}\right| \\
& \left.+\left(R_{x}^{i}-\sin \alpha H_{3}^{i}(u)+\frac{H_{s}^{i}(u)}{2}(|\sin \alpha|-\sin \alpha)\right)\left|\mathbf{e}_{x} \cdot \mathbf{e}\right|\right]
\end{aligned}
$$

2. In the second quadrant (azimuthal angle $\pi / 2 \leq \phi \leq \pi$ )

$$
\begin{aligned}
\left\langle\frac{d R^{i}}{d u}\right\rangle & =\bar{R} \frac{2 \bar{\rho}}{\rho(0)} \frac{t}{2 \pi} T(u)\left[\left(R_{z}^{i}(u)-\cos \alpha H_{1}^{i}(u)\right)\left|\mathbf{e}_{z} \cdot \mathbf{e}\right|\right. \\
& +\left(R_{y}^{i}+\cos \alpha H_{2}^{i}(u)+\frac{H_{c}^{i}(u)}{2}(|\cos \alpha|-\cos \alpha)\right)\left|\mathbf{e}_{y} \cdot \mathbf{e}\right| \\
& \left.+\left(R_{x}^{i}+\sin \alpha H_{3}^{i}(u)+\frac{H_{s}^{i}(u)}{2}(|\sin \alpha|+\sin \alpha)\right)\left|\mathbf{e}_{x} \cdot \mathbf{e}\right|\right]
\end{aligned}
$$

3. In the third quadrant (azimuthal angle $\pi \leq \phi \leq 3 \pi / 2$ ).

$$
\begin{aligned}
\left\langle\frac{d R^{i}}{d u}\right\rangle & =\bar{R} \frac{2 \bar{\rho}}{\rho(0)} \frac{t}{2 \pi} T(u)\left[\left(R_{z}^{i}(u)-\cos \alpha H_{1}^{i}(u)\right)\left|\mathbf{e}_{z} \cdot \mathbf{e}\right|\right. \\
& +\left(R_{y}^{i}-\cos \alpha H_{2}^{i}(u)+\frac{H_{c}^{i}(u)}{2}(|\cos \alpha|-\cos \alpha)\right)\left|\mathbf{e}_{y} \cdot \mathbf{e}\right| \\
& \left.+\left(R_{x}^{i}+\sin \alpha H_{3}^{i}(u)+\frac{H_{s}^{i}(u)}{2}(|\sin \alpha|+\sin \alpha)\right)\left|\mathbf{e}_{x} \cdot \mathbf{e}\right|\right]
\end{aligned}
$$

4. In the fourth quadrant (azimuthal angle $3 \pi / 2 \leq \phi \leq 2 \pi$ )

$$
\begin{aligned}
\left\langle\frac{d R^{i}}{d u}\right\rangle & =\bar{R} \frac{2 \bar{\rho}}{\rho(0)} \frac{t}{2 \pi} T(u)\left[\left(R_{z}^{i}(u)-\cos \alpha H_{1}^{i}(u)\right)\left|\mathbf{e}_{z} \cdot \mathbf{e}\right|\right. \\
& +\left(R_{y}^{i}-\cos \alpha H_{2}^{i}(u)+\frac{H_{c}^{i}(u)}{2}(|\cos \alpha|-\cos \alpha)\right)\left|\mathbf{e}_{y} \cdot \mathbf{e}\right| \\
& \left.+\left(R_{x}^{i}-\sin \alpha H_{3}^{i}(u)+\frac{H_{s}^{i}(u)}{2}(|\sin \alpha|-\sin \alpha)\right)\left|\mathbf{e}_{x} \cdot \mathbf{e}\right|\right]
\end{aligned}
$$

where $i=u, d$

By the reasoning given above, if one mechanism is dominant, the parameters $R_{x}, R_{y}, R_{z}, H_{1}, H_{2}, H_{3}, H_{c}, H_{s}$ for both directions $u$ and $d$ depend only on $\mu_{r}$ and $a$. They are all independent of the other SUSY parameters. 


\section{The Total Event Rates}

We will distinguish two possibilities, namely the directional and the non directional case. Integrating Eq. (33) in the case of caustic rings we obtain for the total non directional rate

$$
\left.R=\bar{R} t \frac{2 \bar{\rho}}{\rho(0)}\left[1-h\left(a, Q_{\min }\right) \cos \alpha\right)\right]
$$

to be compared with the corresponding one for isothermal models:

$$
\left.R=\bar{R} t \frac{\bar{\rho}^{\prime}(0)}{\rho(0)}\left[1+h\left(a, Q_{\min }\right) \cos \alpha\right)\right]
$$

In the above expressions $Q_{\min }$ is the energy transfer cutoff imposed by the detector. The modulation is described by the parameter $h$ only.

The effect of folding with LSP velocity on the total rate is taken into account via the quantity $t$. The SUSY parameters have been absorbed in $\bar{R}$. From our discussion in the case of differential rate it is clear that strictly speaking the quantities $t$ and $h$ also depend on the SUSY parameters. They do not depend on them, however, if one considers the scalar, spin etc. modes separately.

Let us now examine the directional rate. Integrating Eqs. (43) - (46) we obtain:

1. In the first quadrant (azimuthal angle $0 \leq \phi \leq \pi / 2$ ).

$$
\begin{aligned}
R_{d i r}^{i} & =\bar{R} \frac{2 \bar{\rho}}{\rho(0)} \frac{t}{2 \pi}\left[\left(r_{z}^{i}-\cos \alpha h_{1}^{i}\right)\left|\mathbf{e}_{z} \cdot \mathbf{e}\right|\right. \\
& +\left(r_{y}^{i}+\cos \alpha h_{2}^{i}+\frac{h_{c}^{i}}{2}(|\cos \alpha|+\cos \alpha)\right)\left|\mathbf{e}_{y} \cdot \mathbf{e}\right| \\
& \left.+\left(r_{x}^{i}-\sin \alpha h_{3}^{i}+\frac{h_{s}^{i}}{2}(|\sin \alpha|-\sin \alpha)\right)\left|\mathbf{e}_{x} \cdot \mathbf{e}\right|\right]
\end{aligned}
$$

2. In the second quadrant (azimuthal angle $\pi / 2 \leq \phi \leq \pi$ )

$$
\begin{aligned}
R_{d i r}^{i} & =\bar{R} \frac{2 \bar{\rho}}{\rho(0)} \frac{t}{2 \pi}\left[\left(r_{z}^{i}-\cos \alpha h_{1}^{i}\right)\left|\mathbf{e}_{z} \cdot \mathbf{e}\right|\right. \\
& +\left(r_{y}^{i}+\cos \alpha h_{2}^{i}(u)+\frac{h_{c}^{i}}{2}(|\cos \alpha|-\cos \alpha)\right)\left|\mathbf{e}_{y} \cdot \mathbf{e}\right| \\
& \left.+\left(r_{x}^{i}+\sin \alpha h_{3}^{i}+\frac{h_{s}^{i}}{2}(|\sin \alpha|+\sin \alpha)\right)\left|\mathbf{e}_{x} \cdot \mathbf{e}\right|\right]
\end{aligned}
$$

3. In the third quadrant (azimuthal angle $\pi \leq \phi \leq 3 \pi / 2$ ). 


$$
\begin{aligned}
R_{d i r}^{i} & =\bar{R} \frac{2 \bar{\rho}}{\rho(0)} \frac{t}{2 \pi}\left[\left(r_{z}^{i}-\cos \alpha h_{1}^{i}\right)\left|\mathbf{e}_{z} \cdot \mathbf{e}\right|\right. \\
& +\left(r_{y}^{i}-\cos \alpha h_{2}^{i}(u)+\frac{h_{c}^{i}(u)}{2}(|\cos \alpha|-\cos \alpha)\right)\left|\mathbf{e}_{y} \cdot \mathbf{e}\right| \\
& \left.+\left(r_{x}^{i}+\sin \alpha H_{3}^{i}+\frac{h_{s}^{i}}{2}(|\sin \alpha|+\sin \alpha)\right)\left|\mathbf{e}_{x} \cdot \mathbf{e}\right|\right]
\end{aligned}
$$

4. In the fourth quadrant (azimuthal angle $3 \pi / 2 \leq \phi \leq 2 \pi$ )

$$
\begin{aligned}
R_{d i r}^{i} & =\bar{R} \frac{2 \bar{\rho}}{\rho(0)} \frac{t}{2 \pi}\left[\left(r_{z}^{i}-\cos \alpha h_{1}^{i}\right)\left|\mathbf{e}_{z} \cdot \mathbf{e}\right|\right. \\
& +\left(r_{y}^{i}-\cos \alpha h_{2}^{i}+\frac{h_{c}^{i}}{2}(|\cos \alpha|-\cos \alpha)\right)\left|\mathbf{e}_{y} \cdot \mathbf{e}\right| \\
& \left.+\left(r_{x}^{i}-\sin \alpha h_{3}^{i}+\frac{h_{s}^{i}}{2}(|\sin \alpha|-\sin \alpha)\right)\left|\mathbf{e}_{x} \cdot \mathbf{e}\right|\right]
\end{aligned}
$$

\section{Discussion of the Results}

We have calculated the differential as well as the total event rates (directional and non directional) for elastic LSP-nucleus scattering using realistic nuclear form factors. We focused our attention on those aspects of the problem, which do not depend on the parameters of supersymmetry other than the LSP mass. The parameter $\bar{R}$, normally calculated in SUSY theories, was ngt calculated in this work. The interested reader is referred to the literature 15,20 and, in our notation, to our previous work $12,1336$.

We specialized our results for the target ${ }^{127} I$. We considered the effects of the detector energy cutoffs, by studying two typical cases $Q_{\min }=10,20$ $\mathrm{KeV}$. Only the coherent mode due to the scalar interaction was considered. The spin contribution will appear elsewhere.

Special attention was paid to the modulation effect due to the annual motion of the earth. We assumed that the LSP density in our vicinity and the velocity spectrum is that of caustic rings resulting from the self- similar model of Sikivie et al 32 .

We will primarily concentrate on the total rates, which are described in terms of the quantities $t, r_{x}^{i}, r_{y}^{i}, r_{z}^{i}$ for the unmodulated amplitude and $h, h_{1}^{i}, h_{2}^{i}, h_{3}^{i}, h_{c}^{i}, h_{s}^{i}$ for the modulated one. In TABLE II we show how these quantities vary with the detector energy cutoff and the LSP mass. Of the above list only the quantities $t$ and $h$ enter the non directional rate. We notice that the usual modulation amplitude $h$ is small. The main reason is that there are cancelations among the various rings, since some rings are characterized 
by $y_{n z}>1$, while for some others $y_{n z}<1$ (see TABLE I). Such cancelations are less pronounced in the isothermal models. As expected, the parameter t, which contains the effect of the nuclear form factor and the LSP velocity dependence, decreases as the reduced mass increases.

We observe that the quantities $h, r_{j}^{i}, h_{j}^{i}, i=u, d$ and $j=x, y, z, c, s$, being the ratio of two amplitudes, are here essentially independent of the energy cutoff $Q_{\text {min }}$. On the other hand the quantity $t$ decreases after $Q_{\min }$ is introduced, since an important part of the phase space is excluded.

We notice that, unlike the isothermal models, the maximum of modulation occurs around the 2nd of December, something already noticed by Sikivie et al 32 .

Let us now examine the differential rates. We will begin with non-directional one, which is described in terms of the functions $T(u)$ and $H(u)$. These are shown for various LSP masses and $Q_{\min }$ in Fig. 1a for $T(u)$ and Fig. 1b for $H(u)$. We remind the reader that the dimensionless quantity $\mathrm{u}$ is related to the energy transfer Q via Eq. (14) with $Q_{0}=60 \mathrm{KeV}$ for ${ }^{127} I$. Note that, due to our normalization of $\mathrm{T}$, the area under the corresponding curve is unity. This normalization was adopted to bring the various graphs on scale, since the absolute values may vary substantially as a function of the reduced mass. We observe that the function $T(u)$ differs somewhat from the predictions of the isothermal models models. Here the function begins with a maximum at $u=0.0$, while in the isothermal models the maximum occurs at $u=0.1$. Furthermore this function shows less of a reduction as the reduced mass increases (see Fig 1a). The functions $H(u)$ exhibit step behavior in some regions. This is not unexpected, since the LSP velocity spectrum was assumed to be discreet in the Sikivie model.

The directional differential rates, which is now beginning to look like a feasible experimental possibility 2 , is described by the functions

$$
R_{x}, R_{y}, R_{z}, H_{1}, H_{2}, H_{3}, H_{c}, H_{s}
$$

(for both directions $u$ and $d$ ) Due to lack of space we are not going to show the here. We only mention that they were defined as ratios of functions, with $T(u)$ in the denominator. With this definition they are essentially independent of $u$, with values approximately equal to their corresponding total values shown in TABLE II.

\section{Conclusions}

In the present paper we have calculated the parameters, which describe the event rates for direct detection of supersymmetric dark matter. We studied, in particular, the directional variation of the rates and the modulation effect.

The needed local density and velocity spectrum of the LSP were taken from the work of Sikivie et al 32 , viewed as a late in-fall of dark matter into our 
galaxy. They were derived in the context of a self-similar model, which yields 40 caustic rings.

We presented our results in a suitable fashion, i.e by separating the rates into two factors. One factor $\bar{R}$, which carries the dependence on the SUSY parameters, not the subject of the present work, and another, which is essentially independent of all SUSY parameters except the LSP mass. The latter depends mainly on the properties of the LSP velocity distribution, the nuclear parameters and the kinematics. The nuclear form factor was taken into account and the effects of the detector energy cut off were also considered. Strictly speaking the obtained results describe the coherent process in the case of ${ }^{127} \mathrm{I}$, but we do not expect large changes, if the axial current is considered.

Our results, in particular the parameters $t$, see TABLE II, indicate that for large reduced mass, the kinematical advantage of $\mu_{r}$ (see Eqs. (6)- (10) is partly lost when the nuclear form factor and the convolution with the velocity distribution are taken into account. Also, if one attempts to extract the LSPnucleon cross section from the data, in order to compare with the predictions of SUSY models, one must take $t$ into account, since, for large reduced mass, $t$ is different from unity.

In the case of the non-directional total event rates we find that the maximum no longer occurs around June 2nd, but about six months later. The difference between the maximum and the minimum is about $4 \%$, a bit smaller than that predicted by the symmetric isothermal models. It is, however, substantially less than $h=0.46$ predicted by asymmetric isothermal velocity distribution 16.27 .

In the case of the directional rate we found that the rates depend on the direction of observation. The biggest rates are obtained, if the observation is made close to the direction of the sun's motion. The directional rates are suppressed compared to the usual non-directional rates by the factor $f_{\text {red }}=$ $\kappa /(2 \pi)$. We find that $\kappa=r_{z}^{u} \simeq 0.7$, if the observation is made in the sun's direction of motion, while $\kappa \simeq 0.3$ in the opposite direction. The modulation is a bit larger than in the non-directional case, but the largest value, $8 \%$, is not obtained along the sun's direction of motion, but in the x-direction (galactocentric direction).

\section{Acknowledgments}

The author would like to thank Professor Pierre Sikivie for bringing to his attention the idea of caustic rings and velocity peaks. He would like to acknowledge partial support of this work by TMR No ERB FMAX-CT96-0090 of the European Union. He would also like to thank the Humboldt Foundation for their award that provided support during the final stages of this work and Professor Faessler for his hospitality in Tuebingen. 


\section{References}

1. E.W. Kolb and M.S. Turner, The Early Universe, Addison, Wesley, Redwood City, CA ,1990.

P.J.E. Peebles, Principles of Physical Cosmology, Princeton University Press, 1993. M.S. Turner, Cosmology:going beyond the big bang, Physics World, September 1996, p. 31.

2. For a recent review see e.g. G. Jungman et al.,Phys. Rep. 267, 195 (1996).

3. G.F. Smoot et al., (COBE data), Astrophys. J. 396 (1992) L1.

4. E. Gawiser and J. Silk,Science 280, 1405 (1988); M.A.K. Gross, R.S. Somerville, J.R. Primack, J. Holtzman and A.A. Klypin, Mon. Not. R. Astron. Soc. 301, 81 (1998).

5. A.G. Riess et al, Astron. J. 116 (1998), 1009.

6. R.S. Somerville, J.R. Primack and S.M. Faber, astro-ph/9806228; Mon. Not. R. Astron. Soc. (in press).

7. Perlmutter, S. et al (1999) Astrophys. J. 517,565; (1997) 483,565 (astroph/9812133).

S. Perlmutter, M.S. Turner and M. White, Phys. Rev. Let. 83, 670 (1999).

8. Cosmological parameters, astro-ph/9904051; Phys. Rep. 333-334 (1990), 619.

9. D.P. Bennett et al., (MACHO collaboration), A binary lensing event toward the LMC: Observations and Dark Matter Implications, Proc. 5th Annual Maryland Conference, edited by S. Holt (1995);

C. Alcock et al., (MACHO collaboration), Phys. Rev. Lett. 74, 2967 (1995).

10. R. Bernabei et al., INFN/AE-98/34, (1998); R. Bernabei et al., Phys. Lett. B 389, 757 (1996).

11. R. Bernabei et al., Phys. Lett. B 424, 195 (1998); B 450, 448 (1999).

12. J.D. Vergados, J. of Phys. G 22, 253 (1996).

13. T.S. Kosmas and J.D. Vergados, Phys. Rev. D 55, 1752 (1997).

14. M. Drees and M.M. Nojiri, Phys. Rev. D 48, 3843 (1993); Phys. Rev. D 47, 4226 (1993).

15. M.W. Goodman and E. Witten, Phys. Rev. D 31, 3059 (1985);

K. Griest, Phys. Rev. Lett 61, 666 (1988); Phys. Rev. D 38, 2357 (1988) ; D 39, 3802 (1989);

J. Ellis, and R.A. Flores, Phys. Lett. B 263, 259 (1991); Phys. Lett B 300, 175 (1993); Nucl. Phys. B 400, 25 (1993);

J. Ellis and L. Roszkowski, Phys. Lett. B 283, 252 (1992).

16. J.D. Vergados, Phys. Rev. Let. 83, 3597 (1999)

17. J.D. Vergados, Phys. Rev. D 62, 0235XX-1 (2000); astro-ph/0001190 
18. A. Bottino et al., Mod. Phys. Lett. A 7, 733 (1992); Phys. Lett. B 265, 57 (1991); Phys. Lett B 402, 113 (1997); hep-ph / 9709222; hep-ph /9710296;

J. Edsjo and P Gondolo, Phys. Rev. D 56, 1789 (1997);

Z. Berezinsky et al., Astroparticle Phys. 5, 1 (1996);

V.A. Bednyakov, H.V. Klapdor-Kleingrothaus and S.G. Kovalenko, Phys. Lett. B 329, 5 (1994).

19. G.L. Kane et al., Phys. Rev. D 49, 6173 (1994);

D.J. Castaňo, E.J. Piard and P. Ramond, Phys. Rev. D 49, 4882 (1994); D.J. Castaňo, Private Communication;

A.H. Chamseddine, R. Arnowitt and P. Nath, Phys. Rev. Lett. 49, 970 (1982); P. Nath, R. Arnowitt and A.H. Chamseddine, Nucl. Phys. B 227, 121 (1983); R. Arnowitt and P. Nath, Mod. Phys. Lett. 10, 1215 (1995); R. Arnowitt and P. Nath, Phys. Rev. Lett. 74, 4952 (1995); R. Arnowitt and P. Nath, Phys. Rev. D 54, 2394 (1996).

20. R. Arnowitt and P. Nath, hep-ph/9701301; hep-ph/9902237;

S.K. Soni and H.A. Weldon, Phys. Lett. B 126, 215 (1983);

V.S. Kapunovsky and J. Louis, Phys. Lett. B 306, 268 (1993);

M. Drees, Phys. Lett B 181, 279 (1986);

P. Nath and R. Arnowitt, Phys. Rev. D 39, 279 (1989);

J.S. Hagelin and S. Kelly, Nucl. Phys. B 342, 95 (1990);

Y. Kamamura, H. Murayama and M. Yamaguchi, Phys. Lett. B 324, 52 (1994);

S. Dimopoulos and H. Georgi, Nucl. Phys. B 206, 387 (1981).

21. H.E. Haber and G.L. Kane, Phys. Rep. 11775 (1985).

22. S.L. Addler, Phys. Rev. D 11 (1975) 3309.

23. T.P. Cheng, Phys. Rev. D 382869 (1988); H -Y. Cheng, Phys. Lett. B 219347 (1989).

24. M.T. Ressell et al., Phys. Rev. D 48, 5519 (1993); M.T. Ressell and D.J. Dean, hep-ph/9702290.

25. V.I. Dimitrov, J. Engel and S. Pittel, Phys. Rev. D 51, R291 (1995).

26. J. Engel, Phys. Lett. B 264, 114 (1991).

27. M.A. Nikolaev and H.V. Klapdor-Kleingrothaus, Z. Phys. A 345, 373 (1993); Phys. Lett 329 B, 5 (1993); Phys. Rev. D 50, 7128 (1995).

28. D. Spergel, Phys. Rev. D 37, 299 (1996).

29. K.N. Buckland, M.J. Lehner, G.E. Masek, in Proc. 1st Int. Workshop on IDM, p. 475, Ed. N.J.C. Spooner, World Scientific, (1997); Proc. 3nd Int. Conf. on Dark Matter in Astro- and part. Phys. (Dark2000), Ed. H.V. Klapdor-Kleingrothaus, Springer Verlag (2000).

30. Copi, C.J., Heo, J. and Krauss, L.M. (1999),

31. A.K. Drukier et al., Phys. Rev. D 33, 3495 (1986); J.I. Collar et al., Phys. Lett B 275, 181 (1992). 
32. P. Sikivie, I. Tkachev and Y. Wang Phys. Rev. Let. 75, 2911 (1995; Phys. Rev. D 56, 1863 (1997)

P. Sikivie, Phys. Let. b 432, 139 (1998); astro-ph/9810286

33. P.F. Smith et al, Phys. Rep. 307, 275 (1999); N. Spooner, Phys.Rep. 307, 253 (1999); J.J. Quenby et al Astropart. Phys. 5, 249 (1996); Phys. Lett. 351, 70 (1995).

34. J.R. Primack, D. Seckel and B. Sadoulet, Ann. Rev. Nucl. Part. Sci. 38, 751 (1988).

35. P.F. Smith and J.D. Lewin, Phys. Rep. 187, 203 (1990).

36. J.D. Vergados and T.S. Kosmas, Physics of Atomic nuclei, Vol. 61, No 7, 1066 (1998) (from Yadernaya Fisika, Vol. 61, No 7, 1166 (1998).

37. P. Ullio and M. Kamioinkowski, Velocity distributions and annualmodulation signatures of weakly interacting massive particles, hep$\mathrm{ph} / 0006183$.

38. P.C. Divari, T.S. Kosmas, J.D. Vergados and L.D. Skouras, Shell Model Calculations for LSP Scattering Off Light Nuclei, to appear in Phys. Rev C.

39. J.D. Vergados, Phys. Rev. D 58, 103001-1 (1998); 
TABLE I.: The velocity parameters $y_{n}^{\prime}=v_{n} / v_{0}, y_{n z}=y_{n z}^{\prime}=v_{n \phi} / v_{0}, y_{n y}=y_{n y}^{\prime}=$ $v_{n z} / v_{0}, y_{n x}=y_{n x}^{\prime}=v_{n r} / v_{0}$ and $y_{n}=\left[\left(y_{n z}-1\right)^{2}+y_{n y}^{2}+y_{n x}^{2}\right]^{1 / 2}$. Also shown are the quantities: $a_{n}$, the caustic rind radii, and $\bar{\rho}_{n}=d_{n} /\left[\sum_{n=1}^{20} d_{n}\right]$. ( For the other definitions see text ).

\begin{tabular}{|c|c|c|c|c|c|c|c|}
\hline $\mathrm{n}$ & $a_{n}(K p c)$ & $y_{n}^{\prime}$ & $y_{n z}$ & $y_{n y}$ & $y_{n x}$ & $y_{n}$ & $\bar{\rho}_{n}$ \\
\hline 1 & 38.0 & 2.818 & 0.636 & \pm 2.750 & 0.000 & 2.773 & 0.0120 \\
\hline 2 & 19.0 & 2.568 & 1.159 & \pm 2.295 & 0.000 & 2.301 & 0.0301 \\
\hline 3 & 13.0 & 2.409 & 1.591 & \pm 1.773 & 0.000 & 1.869 & 0.0601 \\
\hline 4 & 9.7 & 2.273 & 2.000 & \pm 1.091 & 0.000 & 1.480 & 0.1895 \\
\hline 5 & 7.8 & 2.182 & 2.000 & 0.000 & \pm 0.863 & 1.321 & 0.2767 \\
\hline 6 & 6.5 & 2.091 & 1.614 & 0.000 & \pm 1.341 & 1.475 & 0.0872 \\
\hline 7 & 5.6 & 2.023 & 1.318 & 0.000 & \pm 1.500 & 1.533 & 0.0571 \\
\hline 8 & 4.9 & 1.955 & 1.136 & 0.000 & \pm 1.591 & 1.597 & 0.0421 \\
\hline 9 & 4.4 & 1.886 & 0.977 & 0.000 & \pm 1.614 & 1.614 & 0.0331 \\
\hline 10 & 4.0 & 1.818 & 0.864 & 0.000 & \pm 1.614 & 1.619 & 0.0300 \\
\hline 11 & 3.6 & 1.723 & 0.773 & 0.000 & \pm 1.614 & 1.630 & 0.0271 \\
\hline 12 & 3.3 & 1.723 & 0.682 & 0.000 & \pm 1.591 & 1.622 & 0.0241 \\
\hline 13 & 3.1 & 1.619 & 0.614 & 0.000 & \pm 1.568 & 1.615 & 0.0211 \\
\hline 14 & 2.9 & 1.636 & 0.545 & 0.000 & \pm 1.545 & 1.611 & 0.0180 \\
\hline 15 & 2.7 & 1.591 & 0.500 & 0.000 & \pm 1.500 & 1.581 & 0.0180 \\
\hline 16 & 2.5 & 1.545 & 0.454 & 0.000 & \pm 1.477 & 1.575 & 0.0165 \\
\hline 17 & 2.4 & 1.500 & 0.409 & 0.000 & \pm 1.454 & 1.570 & 0.0150 \\
\hline 18 & 2.2 & 1.455 & 0.386 & 0.000 & \pm 1.409 & 1.537 & 0.0150 \\
\hline 19 & 2.1 & 1.432 & 0.364 & 0.000 & \pm 1.386 & 1.525 & 0.0135 \\
\hline 20 & 2.0 & 1.409 & 0.341 & 0.000 & \pm 1.364 & 1.515 & 0.0135 \\
\hline
\end{tabular}


TABLE II.: The quantities $t$ and $h$ entering the total non directional rate in the case of the target ${ }_{53} I^{127}$ for various LSP masses and $Q_{\text {min }}$ in $\mathrm{KeV}$. Also shown are the quantities $r_{j}^{i}, h_{j}^{i}$ $i=u, d$ and $j=x, y, z, c, s$, entering the directional rate for no energy cutoff. For definitions see text.

\begin{tabular}{|c|c|c|c|c|c|c|c|c|}
\hline & & & & LSP & mass & $\mathrm{GeV}$ & & \\
\hline Quantity & $Q_{\min }$ & 10 & 30 & 50 & 80 & 100 & 125 & 250 \\
\hline $\mathrm{t}$ & 0.0 & 1.451 & 1.072 & 0.751 & 0.477 & 0.379 & 0.303 & 0.173 \\
\hline $\mathrm{h}$ & 0.0 & 0.022 & 0.023 & 0.024 & 0.025 & 0.026 & 0.026 & 0.026 \\
\hline$r_{z}^{u}$ & 0.0 & 0.726 & 0.737 & 0.747 & 0.757 & 0.760 & 0.761 & 0.761 \\
\hline$r_{y}^{u}$ & 0.0 & 0.246 & 0.231 & 0.219 & 0.211 & 0.209 & 0.208 & 0.208 \\
\hline$r_{x}^{u}$ & 0.0 & 0.335 & 0.351 & 0.366 & 0.377 & 0.380 & 0.381 & 0.381 \\
\hline$h_{z}^{u}$ & 0.0 & 0.026 & 0.027 & 0.028 & 0.029 & 0.029 & 0.030 & 0.030 \\
\hline$h_{y}^{u}$ & 0.0 & 0.021 & 0.021 & 0.020 & 0.020 & 0.019 & 0.019 & 0.019 \\
\hline$h_{x}^{u}$ & 0.0 & 0.041 & 0.044 & 0.046 & 0.048 & 0.048 & 0.049 & 0.049 \\
\hline$h_{c}^{u}$ & 0.0 & 0.036 & 0.038 & 0.040 & 0.041 & 0.042 & 0.042 & 0.042 \\
\hline$h_{s}^{u}$ & 0.0 & 0.036 & 0.024 & 0.024 & 0.023 & 0.023 & 0.022 & 0.022 \\
\hline$r_{z}^{d}$ & 0.0 & 0.274 & 0.263 & 0.253 & 0.243 & 0.240 & 0.239 & 0.239 \\
\hline$r_{y}^{d}$ & 0.0 & 0.019 & 0.011 & 0.008 & 0.007 & 0.007 & 0.007 & 0.007 \\
\hline$r_{x}^{d}$ & 0.0 & 0.245 & 0.243 & 0.236 & 0.227 & 0.225 & 0.223 & 0.223 \\
\hline$h_{z}^{d}$ & 0.0 & 0.004 & 0.004 & 0.004 & 0.004 & 0.004 & 0.004 & 0.004 \\
\hline$h_{y}^{d}$ & 0.0 & 0.001 & 0.000 & 0.000 & 0.000 & 0.000 & 0.000 & 0.000 \\
\hline$h_{x}^{d}$ & 0.0 & 0.022 & 0.021 & 0.021 & 0.020 & 0.020 & 0.020 & 0.020 \\
\hline$h_{c}^{d}$ & 0.0 & 0.019 & 0.018 & 0.018 & 0.017 & 0.017 & 0.017 & 0.017 \\
\hline$h_{s}^{d}$ & 0.0 & 0.001 & 0.001 & 0.000 & 0.000 & 0.000 & 0.000 & 0.000 \\
\hline $\mathrm{t}$ & 10.0 & 0.000 & 0.226 & 0.356 & 0.265 & 0.224 & 0.172 & 0.098 \\
\hline $\mathrm{h}$ & 10.0 & 0.000 & 0.013 & 0.023 & 0.025 & 0.025 & 0.026 & 0.026 \\
\hline $\mathrm{t}$ & 20.0 & 0.000 & 0.013 & 0.126 & 0.139 & 0.116 & 0.095 & 0.054 \\
\hline $\mathrm{h}$ & 20.0 & 0.000 & 0.005 & 0.017 & 0.024 & 0.025 & 0.026 & 0.026 \\
\hline
\end{tabular}



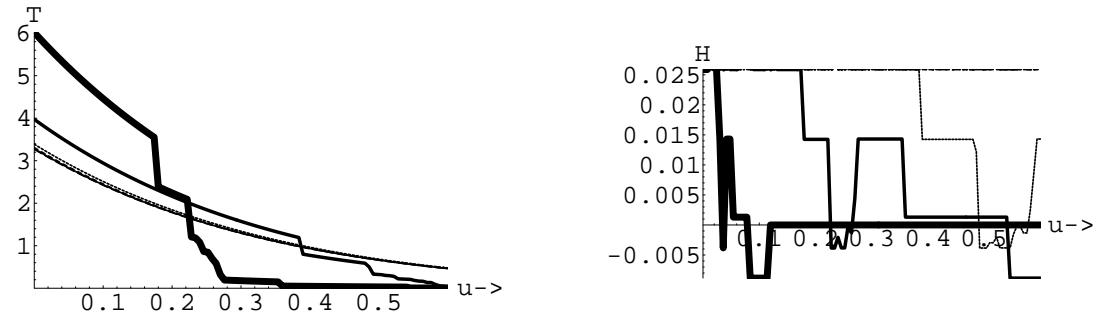

FIG. 1.: The quantities $T(u)$ and $H(u)$ entering the differential amplitude. Thick solid line corresponds to $m_{c h i}=30 \mathrm{GeV}$ the intermediate thickness line to $m_{\chi}=80 \mathrm{GeV}$, the fine line to $m_{\chi}=100 \mathrm{GeV}$. The rest correspond to larger LSP masses and fall on top of each other.f 